\title{
Characterization of host immunity during persistent vaginal colonization by Group B Streptococcus
}

\author{
KA Patras ${ }^{1,4}$, B Rösler ${ }^{1,4}$, ML Thoman ${ }^{2}$ and KS Doran ${ }^{1,3}$
}

Streptococcus agalactiae (Group B Streptococcus, GBS) is a Gram-positive bacterium, which colonizes the vaginal tract in $10-30 \%$ of women. Colonization is transient in nature, and little is known about the host and bacterial factors controlling GBS persistence. Gaining insight into these factors is essential for developing therapeutics to limit maternal GBS carriage and prevent transmission to the susceptible newborn. In this work, we have used human cervical and vaginal epithelial cells, and our established mouse model of GBS vaginal colonization, to characterize key host factors that respond during GBS colonization. We identify a GBS strain that persists beyond a month in the murine vagina, whereas other strains are more readily cleared. Correspondingly, we have detected differential cytokine production in human cell lines after challenge with the persistent strain vs. other GBS strains. We also demonstrate that the persistent strain more readily invades cervical cells compared with vaginal cells, suggesting that GBS may potentially use the cervix as a reservoir to establish long-term colonization. Furthermore, we have identified interleukin-17 production in response to long-term colonization, which is associated with eventual clearance of GBS. We conclude that both GBS strain differences and concurrent host immune responses are crucial in modulating vaginal colonization.

\section{INTRODUCTION}

Streptococcus agalactiae (Group B Streptococcus, GBS) is a Gram-positive bacterium that is frequently isolated from the gastrointestinal and genitourinary tracts of healthy adults, ${ }^{1}$ and has also been isolated from human breast milk. ${ }^{2}$ However, GBS is associated with neonatal invasive disease such as sepsis, pneumonia, and meningitis, affecting $\sim 2,000$ live births per year in the United States alone. ${ }^{3}$ Maternal vaginal colonization rates appear similar in developed and developing countries across all six inhabited continents, ranging from 8 to $18 \%$ with an overall estimated colonization of $12.7 \%{ }^{4,5}$ Current recommendations for neonatal disease prevention consist of late gestation screening and intrapartum antibiotic prophylaxis, and while these practices have reduced early-onset disease, they have had no effect on late-onset disease or maternal colonization. ${ }^{6}$ Alarmingly, GBS adult infections such bacteremia, pneumonia, arthritis, and urinary tract infections are on the rise as well. ${ }^{7,8}$ Furthermore, there is currently no vaccine available for GBS.
Vaginal colonization by GBS may be transient and intermittent, and likely dependent on vaginal $\mathrm{pH}$, normal flora, pregnancy, and estrous cycle, among many other constituents. Increased adherence to vaginal epithelial cells has been observed in vitro as $\mathrm{pH}$ shifts from acidic to neutral; ${ }^{9}$ however, factors that favor persistence of GBS in this complex biological niche are not well understood. GBS determinants that have been shown to contribute to vaginal cell adherence and colonization include the two component system CovRS, surface serine-rich repeat (Srr) proteins, Srr-1 and Srr-2, and pili protein, PilA of GBS Pilus Island-2a. ${ }^{10-12}$ These, and other GBS surface proteins, also facilitate adherence to extracellular matrix constituents such as collagen, ${ }^{13}$ fibrinogen, ${ }^{14}$ fibronectin, ${ }^{15}$ and laminin, ${ }^{16}$ all of which have been identified in vaginal proteome studies, ${ }^{17}$ suggesting potential importance in this environment. Furthermore, GBS possesses metallopeptidases capable of cleaving all four of these extracellular matrix proteins, ${ }^{18}$ which may aid in tissue invasion or niche establishment. Within the vaginal environment, GBS invokes innate immune responses

\footnotetext{
${ }^{1}$ Department of Biology and Center for Microbial Sciences, San Diego State University, San Diego, California, USA. ${ }^{2}$ BioScience Center, San Diego State University, San Diego, California, USA and ${ }^{3}$ Department of Pediatrics, University of California San Diego School of Medicine, La Jolla, California, USA. Correspondence: KS Doran (kdoran@mail.sdsu.edu)

${ }^{4}$ The first two authors contributed equally to this work.
}

Received 23 September 2014; accepted 17 February 2015; published online 8 April 2015. do::10.1038/mi.2015.23 
including neutrophil recruitment ${ }^{10}$ and production of multiple inflammatory cytokines. ${ }^{19} \mathrm{~A}$ recent study found that GBS $\beta$-hemolysin/cytolysin expression is critical for fetal disease and preterm birth in a murine pregnancy model. ${ }^{20}$ Nevertheless, the molecular mechanisms governing GBS vaginal persistence remain to be elucidated.

In this study, we compare the ability of three different GBS strains to colonize the murine vaginal tract, and elicit a host immune response, as well as characterize interactions with human vaginal and cervical epithelial cell lines. Here, for the first time, we examine GBS presence and host immune responses in the cervix and uterus of nonpregnant mice. We further establish key host immune responses including a previously unidentified GBS stimulation of the interleukin-17 (IL-17) immune response and the effect on vaginal persistence. We conclude that both GBS strain differences and concurrent host immune responses are crucial in modulating vaginal colonization.

\section{RESULTS}

\section{Differential persistence of GBS strains within the murine vaginal tract}

We have established a robust murine model of GBS vaginal colonization using a variety of wild-type GBS clinical isolates including A909, ${ }^{10} \mathrm{CJB} 111,{ }^{21}$ and NCTC $10 / 84 .{ }^{11}$ In this model, we have observed transient or intermittent colonization similar to that seen in humans. ${ }^{22}$ Although GBS is not a native murine vaginal species, it is eventually cleared from the vaginal tract in the majority of mice in a range of several weeks to several months. The length of GBS persistence not only depends on mouse strain and duration of $17 \beta$-estradiol treatment (data not shown) but also likely differs among GBS strains. Thus, we examined the ability of different GBS strains representing various serotypes to establish persistent vaginal colonization. Interestingly, GBS strains A909 (serotype Ia) and $\mathrm{COH} 1$ (serotype III) exhibited similar colonization profiles with the majority of mice clearing the bacterium below detection limits in 1-2 weeks, whereas GBS strain, CJB111 (serotype V), persisted beyond several weeks in $>50 \%$ of mice (Figure 1a). Furthermore, although these three strains achieved similar levels of bacterial load within the first 3 days, CJB111 maintained higher bacterial load over time, while A909 and $\mathrm{COH} 1$ both decreased (Figure 1b). At 1 month after inoculation, CJB111 was readily isolated from the vagina, cervix, and uterus, whereas $\mathrm{A} 909$ and $\mathrm{COH} 1$ were not detected (Figure 1c-e).

\section{Differential interaction of GBS strains with human vaginal and cervical epithelial cells}

To gain more insight and to establish that the observed differences between GBS strains observed in murine colonization could be reciprocated in vitro with human cell lines, we performed cellular adherence, invasion, and intracellular survival assays with human vaginal, ectocervical, and endocervical epithelial cell lines. Although we have previously shown that A909 and $\mathrm{COH} 1$ are capable of adhering to and invading these three cell types, ${ }^{12}$ interactions of CJB111 with the female reproductive epithelium have not yet been characterized. For adherence assays, GBS strains were incubated with cells for $2 \mathrm{~h}$, and nonadherent bacteria washed away before quantification. We observed that GBS strains exhibited variable range of ability to attach to vaginal and cervical epithelial cells, with CJB111 being the most adherent strain, whereas A909 was the least adherent overall (Figure 2a-c). We did note increased adherence of $\mathrm{COH} 1$ over A909 to vaginal cells, corresponding with increased vaginal epithelial adherence of serotype III strains over serotype Ia strains noted previously. ${ }^{23}$ Additionally, CJB111 exhibited increased ability to invade certain cell lines compared with $\mathrm{COH} 1$ and A909 (Figure 2d-f). To access invasive capability, we recovered and quantified viable intracellular bacteria from cell lysates after a 2-h infection and a 2-h antibiotic treatment to kill all extracellular bacteria. CJB111 showed significantly increased invasion over $\mathrm{COH} 1$ and $\mathrm{A} 909$ in both vaginal and cervical cell lines (Figure 2d-f). Similarly, for intracellular survival, cell monolayers were infected for $2 \mathrm{~h}$, and then cell lysates analyzed for viable intracellular GBS following antibiotic treatment of extracellular bacteria at indicated time points. Here, CJB111 also exhibited a significantly increased ability to survive within cervical cell lines at all time points tested compared with $\mathrm{COH} 1$ and A909, but no striking differences were observed in vaginal cells (Figure 2g-i). These results highlight that CJB111 acts discretely from $\mathrm{COH} 1$ and A909 in its interaction with host epithelium, consonant with its long-term colonization phenotype in vivo (Figure 1). Furthermore, these results demonstrate that CJB111 more readily invades and/or survives within cervical epithelium, which may be beneficial in niche establishment and long-term cervicovaginal persistence.

\section{GBS cytokine induction in human vaginal and cervical epithelial cells}

We next sought to determine whether CJB111 stimulates a distinct immune profile within the female reproductive tract compared with other GBS strains. Our previous studies with human vaginal epithelial cells using microarray, reverse transcription-PCR, and protein analysis revealed increased transcription and production of multiple proinflammatory cytokines and chemokines including IL-8, CCL20, CXCL1, and CXCL2 following exposure to GBS. ${ }^{10}$ The microarray data also suggested additional innate components such as IL-1 $\beta$, IL-6, IL-23, and IL-36 $\gamma$ were also stimulated in vaginal epithelium in response to GBS. These cytokines have been implicated in adaptive immunity, such as the $\mathrm{T}$ helper type $17\left(\mathrm{~T}_{\mathrm{H}} 17\right)$ cell response, which contributes to neutrophil homeostasis and sustained inflammatory signaling. ${ }^{24,25}$

We sought to verify and extend the earlier microarray data by analyzing transcript abundance in vaginal, ectocervical, and endocervical cells after infection with GBS strains. These experiments revealed IL-1 $\beta$, IL-6, IL-23, and IL-36 $\gamma$ mRNA transcripts were upregulated in all three cell lines compared with media controls (Figure 3a-c). Interestingly, $\mathrm{COH} 1$ and A909 invoked more significant upregulation of IL-36 $\gamma$ in all 
a
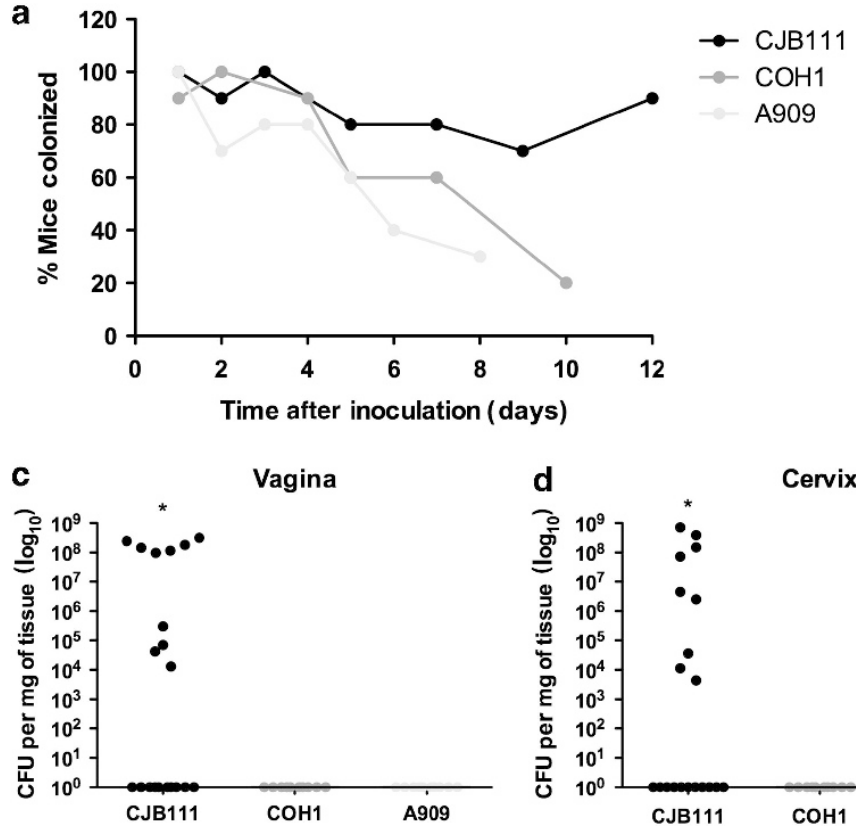
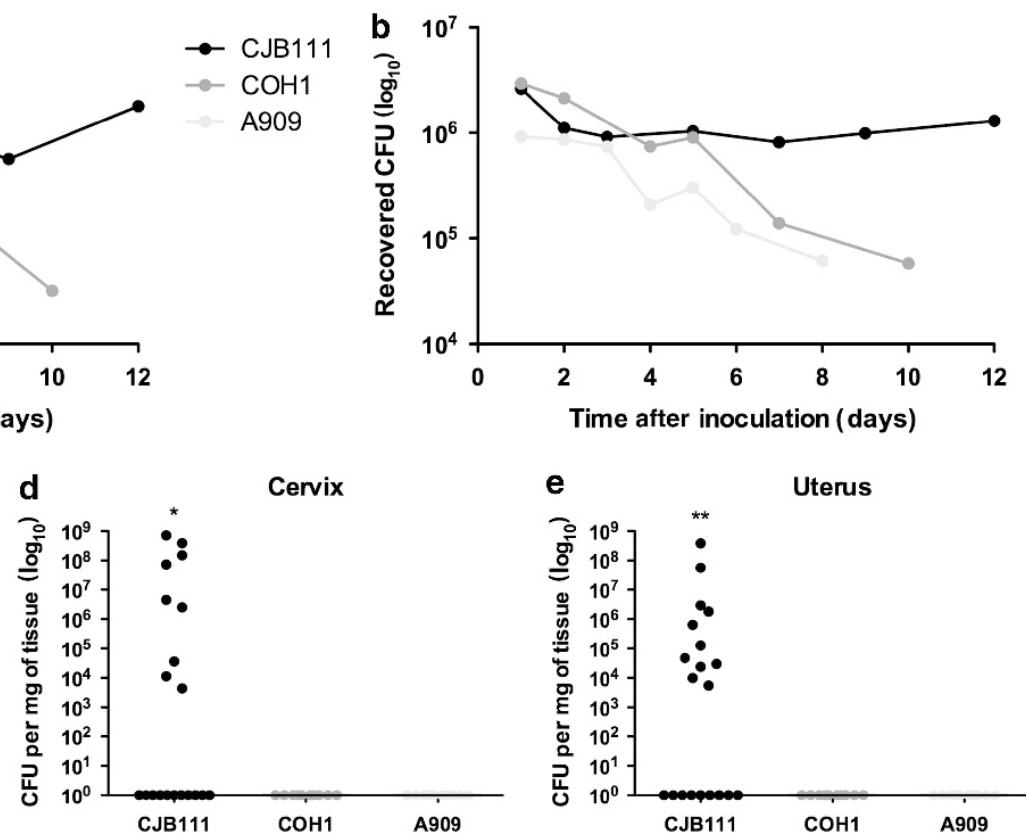

Figure 1 Streptococcus agalactiae (Group B Streptococcus, GBS) strain persistence in the murine vaginal tract. CD1 mice ( $n=10$ per group) were colonized with $1 \times 10^{7} \mathrm{CFU}$ (colony-forming unit) of GBS clinical isolates $\mathrm{CJB111}, \mathrm{COH} 1$, or A909 in the vaginal lumen. GBS persistence was determined by swabbing the vagina and plating recovered bacteria. (a) Percentage of mice with detectable GBS within the vaginal lumen via swabbing. Limit of detection is 50 CFU. (b) Mean recovered GBS CFU of same mice as in a. (c-e) Reproductive tract tissues were isolated 4 weeks after inoculation with GBS strains. Bacterial load was quantified by plating serially diluted tissue homogenates. In vivo experiments were conducted independently at least two times and data from one representative experiment is shown. Data were analyzed using Kruskal-Wallis with Dunn's multiple comparisons post-test. ${ }^{\star} P<0.05 ;{ }^{* \star} P<0.01$.
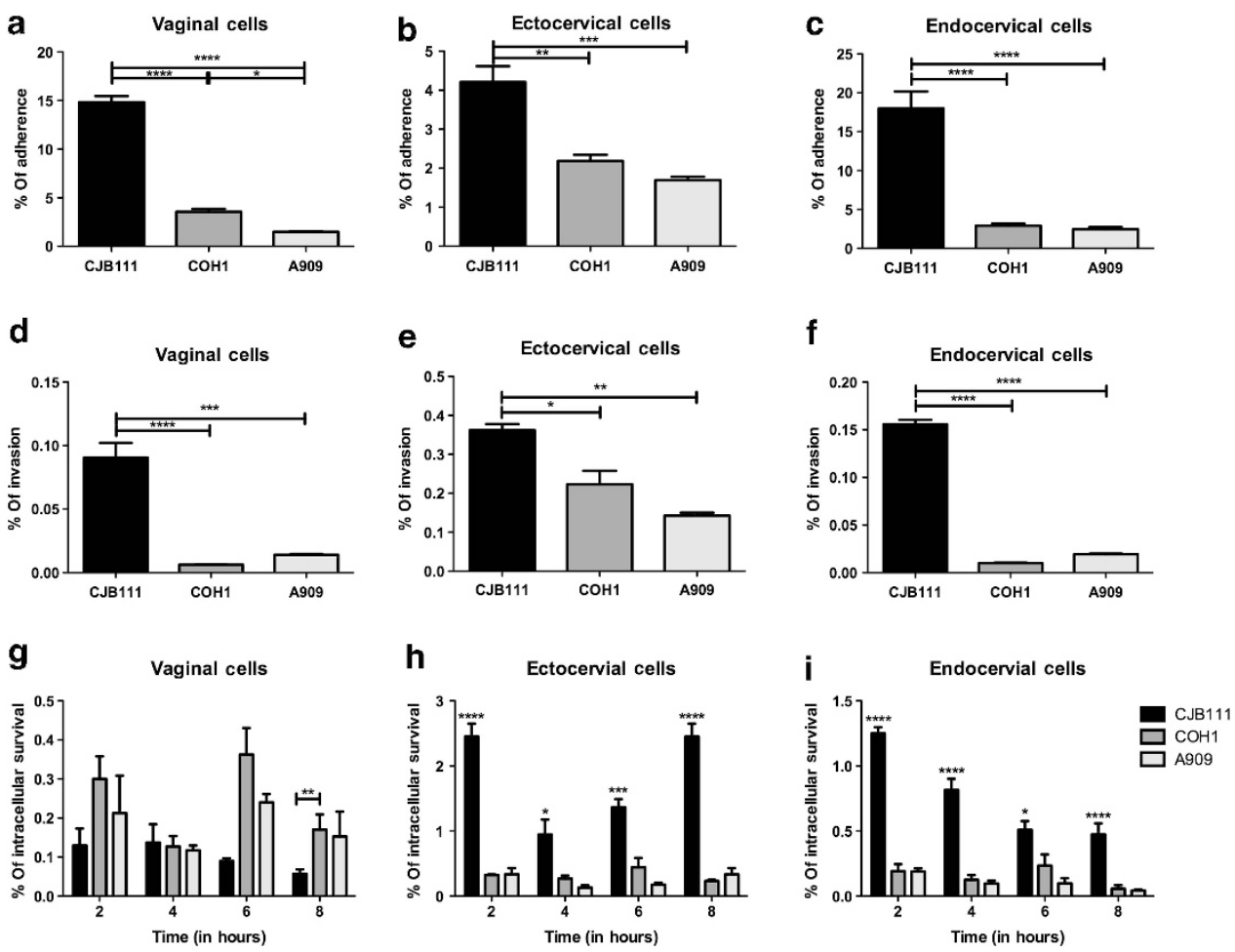

Figure 2 Interaction of Streptococcus agalactiae (Group B Streptococcus, GBS) strains with human female reproductive epithelial cells. (a-c) Adherence, $(\mathbf{d}-\mathbf{f})$ invasion, or $(\mathbf{g}-\mathbf{i})$ intracellular survival of GBS CJB111, $\mathrm{COH} 1$, or A909 with indicated epithelial cells, multiplicity of infection (MOI) = 10 for $\mathbf{a}-\mathbf{f}$ and $\mathrm{MOI}=1$ for $\mathbf{g}-\mathbf{i}$. For $\mathbf{h}-\mathbf{i}$, significance given is $\mathrm{CJB} 111$ in comparison with both $\mathrm{COH} 1$ and $\mathrm{A} 909$. Experiments were repeated at least two times with four replicates and data from a representative experiment is shown. Data were analyzed by one-way analysis of variance (ANOVA) with Bonferroni's multiple comparisons post-test for $\mathbf{a}-\mathbf{f}$ and two-way repeated-measures ANOVA with Bonferroni's multiple comparisons post-test for $\mathbf{g}-\mathbf{i}$. ${ }^{*} P<0.05$; ${ }^{\star *} P<0.01 ;{ }^{* \star *} P<0.001 ;{ }^{* * \star *} P<0.0001$. 
three cell types, IL-23 in vaginal and endocervical cells, IL- $1 \beta$ in vaginal and ectocervical cells, and IL-6 in endocervical cells when compared with stimulation with CJB111. We next examined cytokine production at the protein level to confirm biological relevance. In this study, we observed that IL-1 $\beta$ production significantly increased in all three cell lines in response to $\mathrm{COH} 1$ and $\mathrm{A} 909$ at 6 and $8 \mathrm{~h}$ after infection compared with media controls; however, CJB111 did not illicit this same response (Figure 3d-f). Additionally, we were unable to detect IL-23 under any of the tested conditions (data not shown). We quantified IL-36 $\gamma$ production via western blot, and detected significantly heightened production in ectocervical cells treated with A909 after 8 and $10 \mathrm{~h}$, and in endocervical cells treated with A909 at $6 \mathrm{~h}$ (Figure 3h,i). Although we did note increased IL-36 $\gamma$ production in vaginal cells in response to CJB111 at $6 \mathrm{~h}$ (Figure 3g), we also observed lower levels of intracellular CJB111 in the vaginal cell assays (Figure 2). Taken together, the inverse relationship between cytokine production and intracellular viable CJB111 in the reproductive tract epithelium is of great interest and the topic of future study.

\section{GBS colonization and cytokine production}

Although GBS is a frequent colonizer of both pregnant and nonpregnant healthy women, the human host response to GBS presence within the vaginal tract remains to be fully described. Thus far, this response has been preliminarily characterized using in vitro cell-based assays, ${ }^{10}$ and murine models. ${ }^{10,19}$ To better define early immune responses to GBS strains A909, $\mathrm{COH} 1$, and $\mathrm{CJB} 111$ in vivo within the murine reproductive tract, a multiplexed electrochemiluminescence detection assay was used to ascertain the presence of interferon- $\gamma$, IL-1 $\beta$, IL-2, IL-4, IL-5, IL-6, IL-10, IL-12p70, keratinocyte-derived chemokine/growth-related oncogene alpha $(\mathrm{KC} / \mathrm{GRO} \alpha)$, and tumor necrosis factor- $\alpha$ in the vagina, cervix, and uterus 2 days after inoculation with GBS. We observed stimulation of IL-1 $\beta$, IL-6, and $\mathrm{KC}$, whereas the other cytokines on the panel were
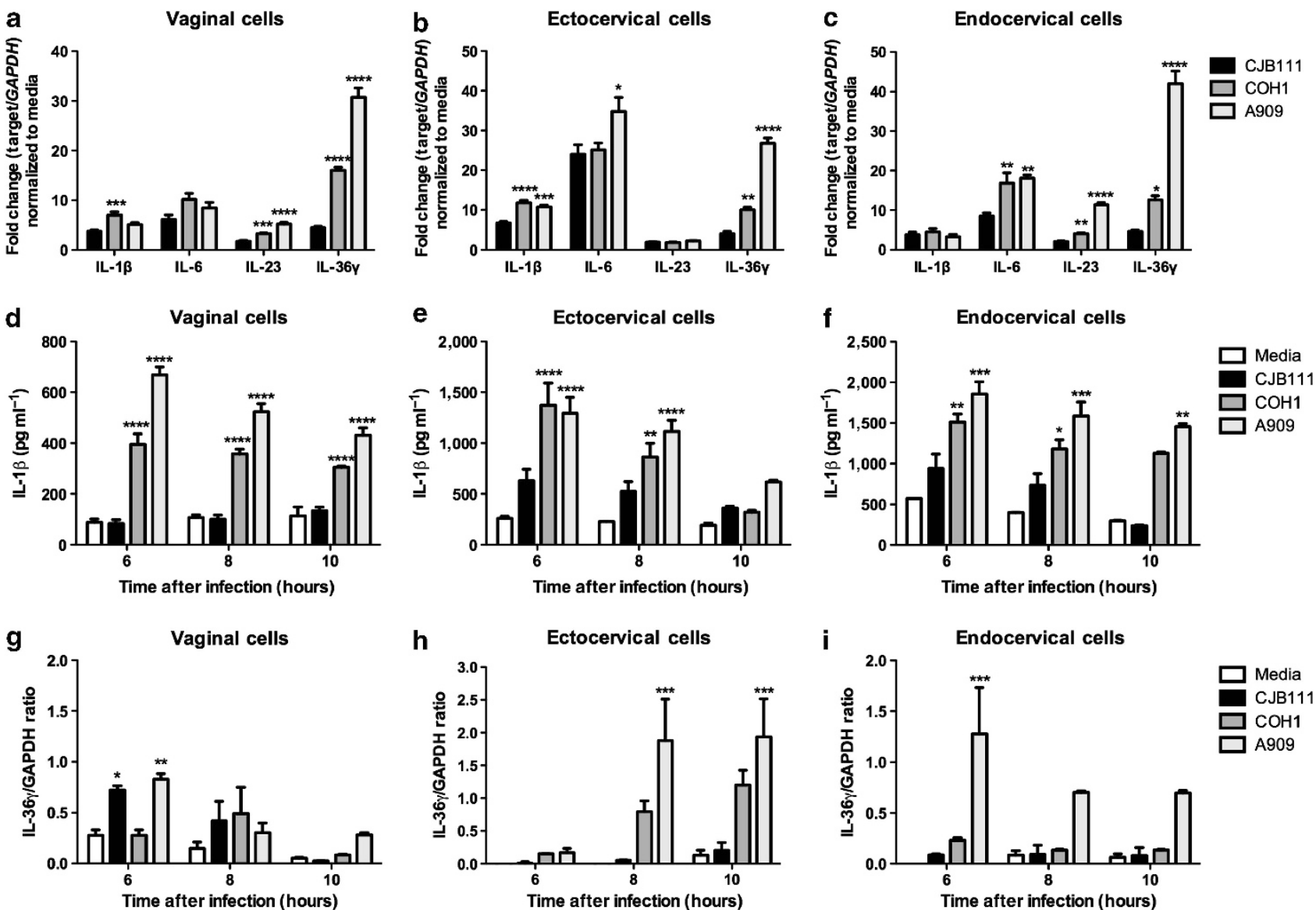

Figure 3 Streptococcus agalactiae (Group B Streptococcus, GBS) cytokine induction in human female reproductive epithelial cells. (a-c) Transcript abundance of interleukin-1 $\beta$ (IL-1 $\beta$ ), IL-6, IL-23, and IL-36 $\gamma$ in human epithelial cells was determined using quantitative reverse transcription-PCR following infection with CJB111, COH1, or A909 (multiplicity of infection $(\mathrm{MOI})=10$ ). Fold change was calculated using glyceraldehyde 3-phosphate dehydrogenase (GAPDH) and then normalized to media controls as described in Methods section. Statistical values are in reference to CJB111. (d-f) Protein expression of IL-1 $\beta$ and $(\mathbf{g}-\mathbf{i})$ IL-36 $\gamma$ in human epithelial cell lysates was determined as described in Methods section $4 \mathrm{~h}$ after infection with CJB111, $\mathrm{COH} 1$, or $\mathrm{A} 909$ at an $\mathrm{MOI}$ of 10 . Statistical values are in reference to media controls. Data are one representative experiment of at least two independent experiments performed in four replicates at minimum. Data were analyzed by one-way analysis of variance (ANOVA) with Bonferroni's multiple comparisons post-test for $\mathbf{a}-\mathbf{c}$ and two-way repeated-measures ANOVA with Bonferroni's multiple comparisons post-test for (d)-(i). ${ }^{*} P<0.05$; ${ }^{\star \star} P<0.01 ;{ }^{* \star \star} P<0.001 ;{ }^{\star \star \star \star} P<0.0001$. 
detected at much lower levels (data not shown). To confirm this preliminary screen, mice were colonized with CJB111, COH1, and A909, and 2 days after inoculation, we recovered reproductive tract tissues for protein analysis and bacterial quantification. In vaginal tissues, GBS colonization resulted in significantly higher levels of KC and IL- 6 , and elevated levels of IL- $1 \beta$ and macrophage inflammatory protein-2 (MIP-2), but no increased production of IL-23 (Figure 4a-e). No GBS strain differences in cytokine profiles were noted upon in vivo infection in contrast to in vitro results. When bacterial loads were quantified from these same mouse tissues, more CJB111 colony-forming units (CFUs) were recovered than the other strains (Figure $\mathbf{4 g - i}$ ), particularly in the cervix, suggesting that although CJB111 stimulates the host immune response similarly to other GBS strains, it possesses a unique set of factors allowing it to thrive within this environment to promote longer vaginal persistence. Given that several early cytokines linked to the $\mathrm{T}_{\mathrm{H}} 17$ response were present in the murine reproductive tract, we measured IL-17A production after 4 weeks of colonization to allow time for development of an adaptive immune response. Interestingly, we observed significantly more IL-17A in mice colonized with CJB111 compared with phosphate-buffered saline (PBS) controls, but not in mice colonized with $\mathrm{COH} 1$ or A909 (Figure 4f). Additionally, we analyzed IL-17A levels at days 2, 5, and 10 after inoculation with A909, COH1, or CJB111 and did not observe any increase in IL17A production over PBS controls at these earlier time points (data not shown).

\section{Presence of IL-17 within the reproductive tract is associated with reduced clearance of the persistent GBS strain CJB111}

Several groups have demonstrated that the $\mathrm{T}_{\mathrm{H}} 17$ response is activated in response to Candida albicans ${ }^{26}$ and Neisseria gonorrhoea $e^{27}$ within the vaginal tract, and one group identified elevated, but not significant, IL-17 in the total reproductive tract of GBS-colonized mice. ${ }^{19}$ However, the role of IL-17A production in response to GBS pathogenesis or colonization has not yet been recognized.
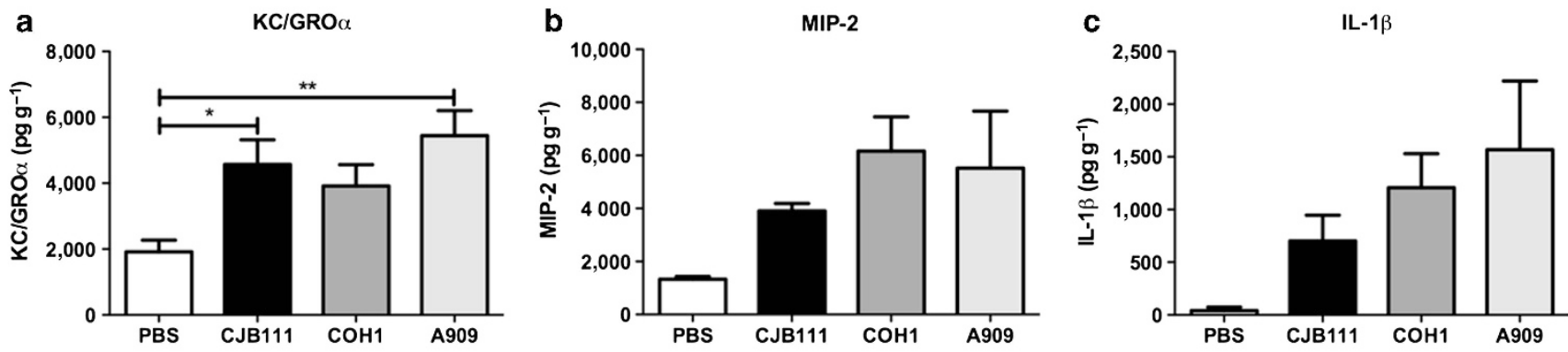

d

e

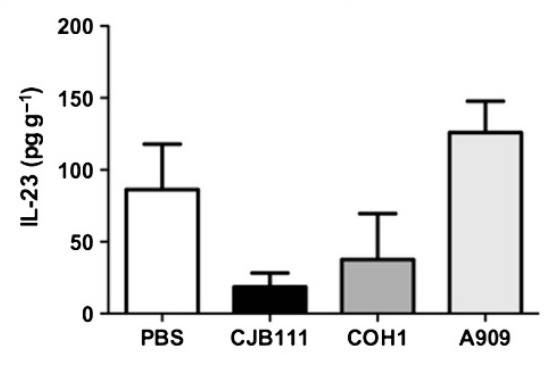

h

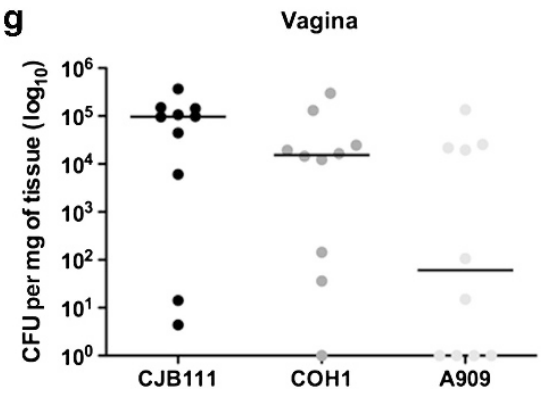

IL-6
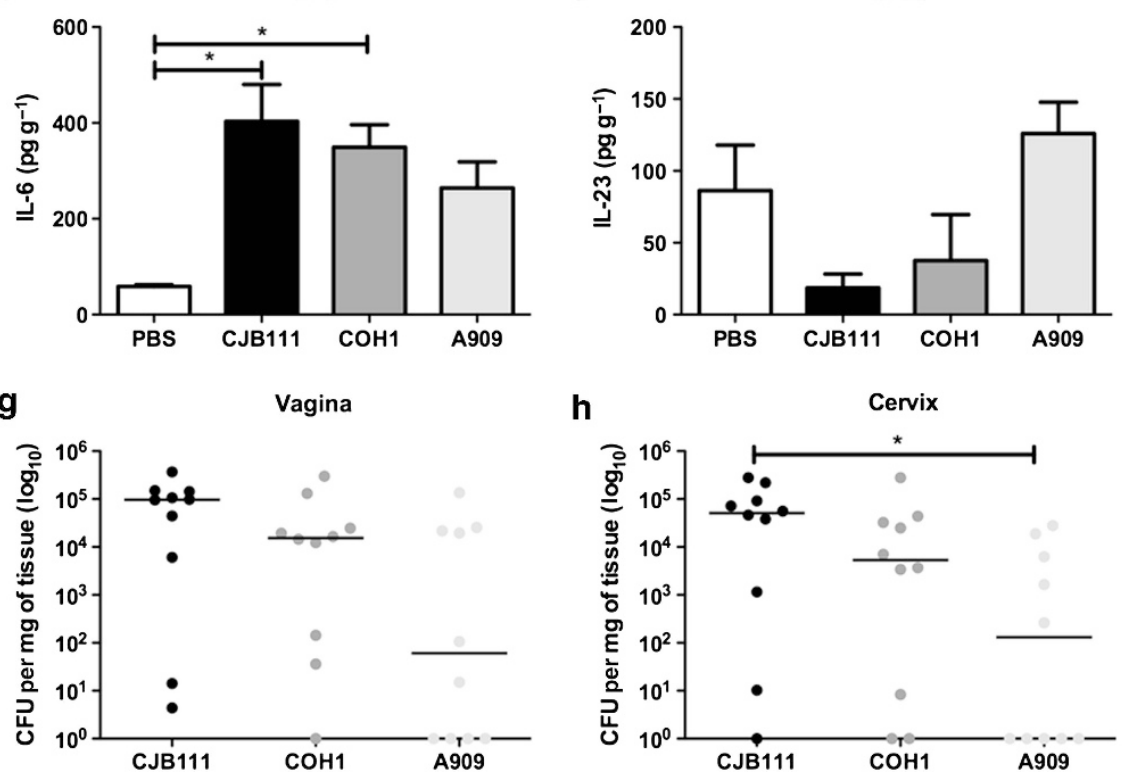

f

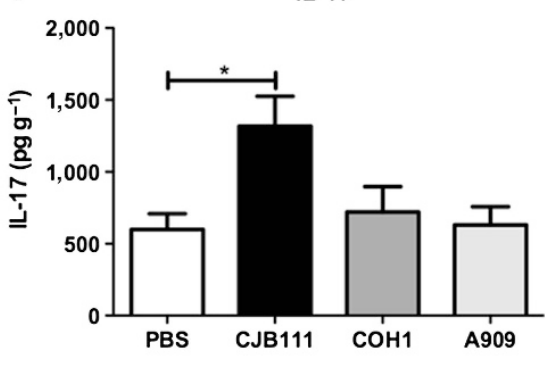

i

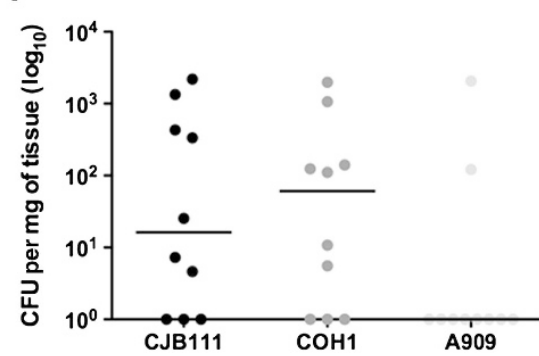

Figure 4 Streptococcus agalactiae (Group B Streptococcus, GBS) colonization and cytokine production in the murine reproductive tract. (a) Keratinocyte-derived chemokine/growth-related oncogene alpha $(\mathrm{KC} / \mathrm{GRO} \alpha)$, (b) macrophage inflammatory protein-2 (MIP-2), (c) interleukin-1 $\beta$ (IL-1 $\beta$ ), (d) IL-6, and (e) IL-23 levels in vaginal homogenates from mice 2 days after inoculation, or (f) IL-17A levels at 4 weeks after inoculation, were quantified by enzyme-linked immunosorbent assay (ELISA). Data are the combined results of two independent experiments ( $n=10-20$ per group). (g-i) GBS load in reproductive tract tissue homogenates collected from mice 2 days after inoculation ( $n=10$ per group). Lines represent median values of each group. Data were analyzed by Kruskal-Wallis with Dunn's multiple comparisons post-test. ${ }^{\star} P<0.05$; ${ }^{\star \star} P<0.01$. 
Consistent with the production of IL-17A in the reproductive tract after 4 weeks of GBS colonization, we observed the presence of IL-17 + cells beneath the epithelium, within the lamina propria, in both vaginal and uterine tissues (Figure 5i, j). To determine the outcome of IL-17A production during long-term GBS colonization, we colonized mice with CJB111 and collected tissues at 2 and 4 weeks after inoculation for bacterial quantification and evaluation of IL-17A + cells. We separated mice into two groups to analyze the data: mice that remained colonized at the time of killing, and mice that had cleared CJB111 to below our limit of detection. By 2 weeks after inoculation, mice that had cleared CJB111 possessed significantly higher abundance of total IL-17A + cell populations in the vagina $(3.1 \%)$ than colonized mice $(0.98 \%$, $P=0.0320)$. This same trend of increased total IL-17A + cells was present in the cervix $(P=0.0666)$ and uterus $(P=0.0979)$ (Figure 5a). The average basal levels of total IL-17A + cells in uninfected mice were $0.8 \%$ in the vagina, $0.6 \%$ in the cervix, and $1.1 \%$ in the uterus. We further identified that IL-17A + cells expressed surface markers Ly6G, CD11b, or CD4, and observed increased levels in cleared mice in all tissues, but particularly in Ly6G +/IL-17+ and CD11b +/IL-17 + cells the uterus (Figure 5b-d). At 4 weeks after inoculation, we determined that the primary location of IL-17 + cells was in the cervix, with distinctive differences between colonized mice and those that had cleared GBS. Interestingly, of these IL-17+ cells, those expressing either Ly6G or CD4 were significantly elevated in the cervix of cleared mice vs. those that remained colonized (Figure 5g). This effect was not seen in the vagina or uterus, both of which contained much lower quantities of total IL-17A + cells (Figure 5e,f,h).

To test whether IL-17A presence within the vagina is sufficient to result in successful GBS clearance, we colonized mice with CJB111 for $24 \mathrm{~h}$, and then began daily vaginal administration of recombinant IL-17 (rIL-17) as described in the Methods section. We found that after 2 days of rIL-17 treatment, treated mice had significantly lower bacterial load than nontreated controls (Figure 5k). Taken together, these
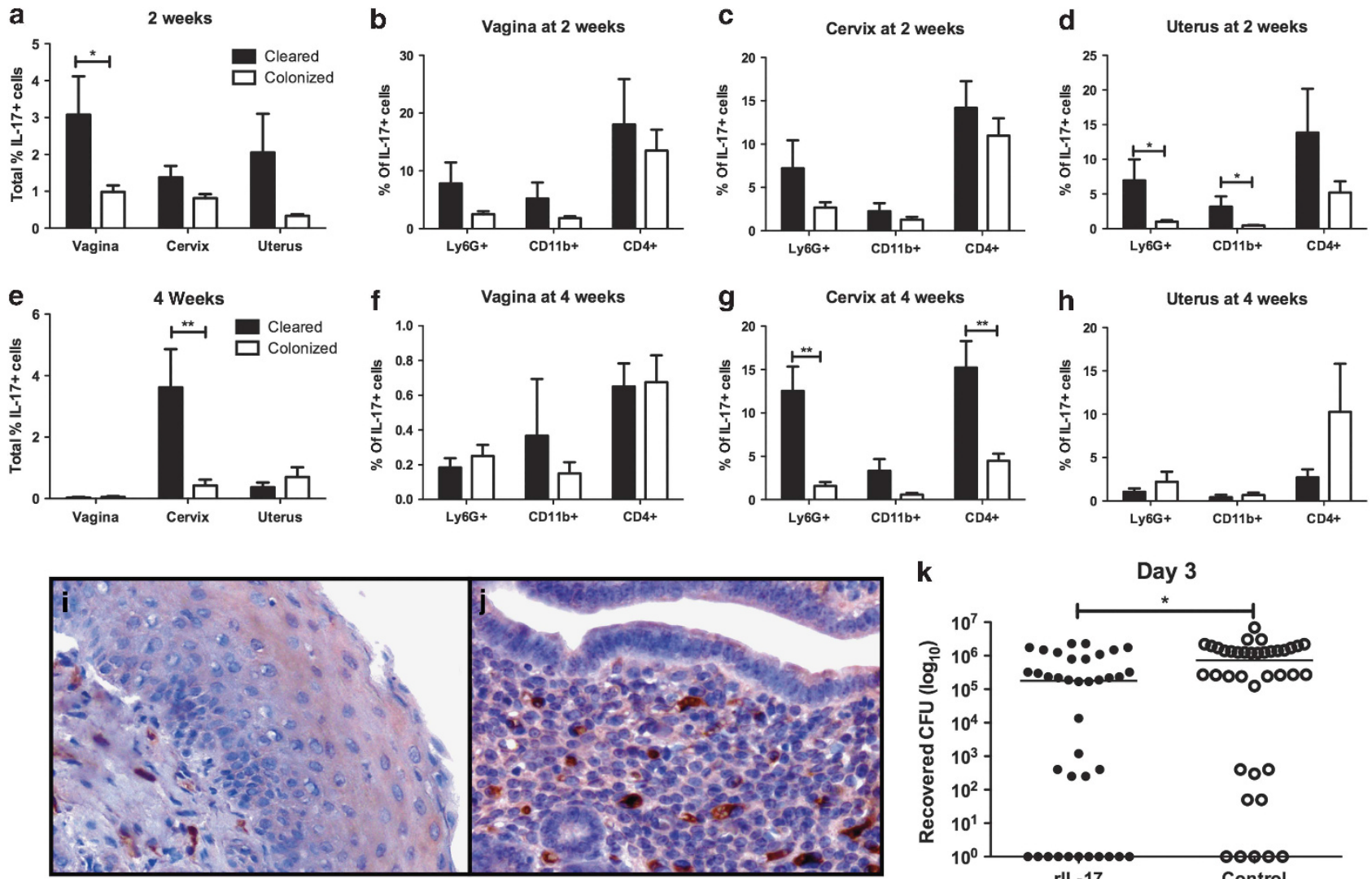

$\mathbf{k}$

Day 3

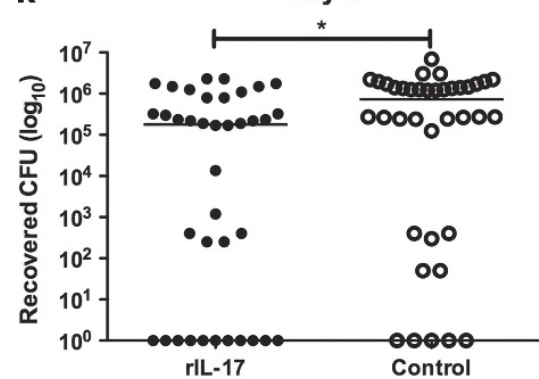

Figure 5 Presence of interleukin-17 (IL-17) within the reproductive tract is associated with reduced clearance of Streptococcus agalactiae (Group B Streptococcus, GBS) CJB111. (a and e) Total IL-17A + cells present in reproductive tract tissues collected from mice ( $n=4-6$ per group) at indicated time points after inoculation with CJB111 were quantified by flow cytometry as described in Methods section. Cleared mice (black bars) were separated from colonized mice (white bars) if GBS counts were below the limit of detection at the time of killing (50 CFU per tissue). (b-d and $\mathbf{f}-\mathbf{h}$ ) Surface markers Ly6G, CD11b, and CD4 present on populations identified within total IL-17A + cells from a and $\mathbf{e}$. Representative data from one of two independent experiments is shown. Immunohistochemistry of the (i) vagina and (j) uterus of GBS-colonized mice with IL-17 + cells visualized with diaminobenzidine (brown) and counterstained with hematoxylin (blue). Original magnification, $\times 200$. (k) Mice were colonized with CJB111, and 1 day later, received daily treatment of recombinant IL-17 (rIL-17) within the vaginal lumen. Data shown are from 3 days after inoculation with CJB111, and is the result of three independent experiments combined (total $n=38$ per group). Lines represent median values of each group. Data were analyzed by Mann-Whitney. ${ }^{\star} P<0.05$; ${ }^{\star *} P<0.01$ 
experiments suggest that in mice colonized with CJB111, a more persistent strain of GBS, production of IL-17A corresponds with the eventual ability to clear GBS.

\section{DISCUSSION}

GBS continues to be a leading cause of neonatal disease including sepsis and meningitis in many developed nations, ${ }^{2}$ yet the scope of our understanding of host response to GBS vaginal colonization, and subsequent preventative measures to control vertical transmission of GBS from mother to newborn, remain limited. In this study, we observed two distinct factors controlling GBS interactions with the female reproductive tract: variable determinants present among GBS strains and host immune profiles. GBS strains displayed differential abilities to adhere to and survive intracellularly among multiple epithelial cell types, as well as varying lengths of vaginal persistence. Concurrently, increased host innate cytokine production corresponded to decreased intracellular GBS in vitro, in line with increased IL-17 + cell populations in mice that had successfully cleared GBS. These data substantiate that both GBS strain differences and concurrent host immune responses are crucial in modulating vaginal colonization.

One of the most widely recognized GBS virulence factors, the polysaccharide capsule, has also been used to separate GBS strains into 10 unique serotypes based on the chemical structure (Ia, Ib, II, III, IV, V, VI, VII, VIII, and IX). ${ }^{28}$ Epidemiologic studies have revealed that serotype III strains are significantly more prevalent in neonatal meningitis, ${ }^{29}$ whereas serotype $\mathrm{V}$ is dominant in adult cases of GBS infections, with serotypes Ia, Ib, II, III, and V representing the vast majority of both neonatal and adult cases. ${ }^{7}$ Elements driving the prevalence of serotypes in various disease states are currently unknown, but likely depend on bacterial virulence and fitness factors common within serotypes as well as concurrent host responses. In this study, we characterized distinct phenotypes of strains representing serotypes Ia, III, and V, with the serotype V strain CJB111 displaying greater intracellular survival and lesser cytokine stimulation in cervical cells, and increased persistence in the mouse vaginal tract. Although all three of these strains have been fully or partially sequenced, ${ }^{30}$ the molecular machinery determining the length of GBS vaginal colonization is unknown. Possible explanations for this phenomenon include either differences in their repertoire of cellular adhesins/ invasins, ability to outcompete normal flora, and/or dialog with the host immune system. CJB111 possesses a set of 20 unique genes compared with other fully sequenced GBS strains, ${ }^{30}$ and is adept at forming biofilms in vitro. ${ }^{31}$ Others have previously noted serotype V strain antigens (from CJB111) invoke an alternative IgM immune response compared with other serotypes, which conjure an IgG response. ${ }^{32}$ Future work seeks to identify the genetic constituents of CJB111 that allow for increased perpetuation within the host, both intracellularly and at the mucosal surface.

Another aim of this study was to expand our understanding of female reproductive tract responses to GBS. Clinical studies have demonstrated that vaginal IL- $1 \alpha$ levels correlate to GBS colonization status in nonpregnant women, ${ }^{33}$ whereas increased levels of maternal serum IL-1 $\beta$ are associated with increased risk of GBS infection and early term birth in pregnancy. ${ }^{34}$ Using murine models of GBS, we have previously observed increased vaginal cytokine levels of MIP-2 and IL-1 $\beta$ in response to hyperhemolytic GBS, ${ }^{10}$ and another group has noted increased reproductive tract levels of tumor necrosis factor- $\alpha$ and IL- $1 \alpha$ after several weeks of GBS colonization. ${ }^{19}$ However, global cytokine changes over time, and the subsequent effect on vaginal colonization, have yet to be established.

We used human vaginal and cervical epithelial cells to examine potential human immune responses to infection with several strains of GBS. Based on earlier microarray data of vaginal cells infected with GBS, we pursued several enhanced innate components: IL-1 $\beta$, IL-6, IL-23, and IL-36 $\gamma$. IL-1 $\beta$ is a potent inflammatory cytokine that in conjunction with IL-6 and TGF- $\beta$ induce $\mathrm{T}_{\mathrm{H}} 17$ cell maturation. ${ }^{35}$ IL-23 promotes $\mathrm{T}_{\mathrm{H}} 17$ cell maintenance and function, but has also been linked to T-cell-independent inflammation in mucosal surfaces. ${ }^{36} \mathrm{IL}-36 \gamma$, a recently identified IL-1 family member produced by internal epithelium and keratinocytes, is a potent inducer of IL-6, and heightens cytokine production of activated CD4 $+\mathrm{T}$ cells. ${ }^{37}$ Past work has identified spontaneous production of IL-6 and IL-8 in these vaginal, ectocervical, and endocervical cells lines, and production of IL-1 $\beta$ upon cytokine stimulation. ${ }^{38}$ We have previously demonstrated GBS induction of IL-8 from vaginal epithelial cells. ${ }^{10}$ In this work, we established that these three cell lines produced IL- $1 \beta$ and IL-36 $\gamma$ protein in response to GBS, but were unable to detect IL-23. A former study carried out with this exact vaginal cell line was also unable to detect IL-23 production either spontaneously or with lactic acid stimulation; ${ }^{39}$ thus, it is possible that these cell lines are incapable of generating functional IL-23. To our knowledge, this is the first recorded incidence of IL-36 $\gamma$ production from these three cells lines. Overall, IL-36 $\gamma$ production in the female reproductive tract in response to pathogens has not been well characterized, with the exception of HIV infection in vaginal cells. ${ }^{40}$

Alternatively in vivo, we observed increased production of several innate immune cytokines in response to A909, COH1, and CJB111 over PBS controls; however, no differences were seen between GBS strains, suggesting that early host responses to GBS are similar independent of strain or serotype. Even so, CJB111 was able to persist beyond these early immune responses in the majority of mice in contrast to A909 and $\mathrm{COH} 1$. A distinctive host immune response we identified in this work was increased production of IL-17A within the vaginal tract of mice persistently colonized with CJB111. The $\mathrm{T}_{\mathrm{H}}$ 17/IL-17 response has been associated with control of mucosal pathogens in multiple host tissues, including the lung $^{41}$ and gut. ${ }^{42} \mathrm{~T}_{\mathrm{H}} 17$ cells and their associated responses are very closely linked to the microbiota living upon the mucosal surface of a given tissue, considering that germ-free mice possess significantly reduced IL-17+ cell populations in the small intestine. ${ }^{43}$ Specifically within the human vaginal tract, detection of at least one dominant Lactobacillus species has 
been associated with increased presence of vaginal IL-17 and vascular endothelial growth factor. ${ }^{44}$ Moreover, other clinical work has revealed that percentages of vaginal Lactobacillus species are reduced in pregnant women that are GBS-positive. ${ }^{45}$ These human studies, combined with our in vivo work here, suggest that vaginal IL-17, stimulated by either healthy normal flora or vaginal inflammation in response to GBS, may be an important immune response for controlling GBS colonization and maintaining a beneficial vaginal microbiota.

In this work, we identified at least two cellular populations that produced reproductive tract IL-17A including neutrophils and $\mathrm{CD} 4+\mathrm{T}$ cells. Whether both sources are effective in reducing GBS in our system remains to be determined. However, previous studies have noted influx of both IL-17A-producing CD4 + T cells and neutrophils in mucosal models of pathogenesis. ${ }^{26,46}$ Additionally, the timing of IL-17A production at the mucosal surface varies across pathogenic agents and host tissues, with responses occurring within 2-3 days or up to 6 weeks in the lung ${ }^{46,47}$ or $\sim 2$ weeks in the vaginal tract. ${ }^{26}$ In our model, we observed higher numbers of IL-17A + cells in the vagina and uterus at 2 weeks, and higher numbers in the cervix at 4 weeks (Figure 5). Interestingly, enzyme-linked immunosorbent assay (ELISA) analysis identified increased IL-17A in the vaginal tract at 4 weeks (Figure 4), even though flow cytometry displayed very low populations of IL-17A + cells at this time point (Figure 5). It is possible that cervical IL-17A + cells were the source of vaginal IL-17A at this time, as cervical cytokines are thought to be critical in protecting against reproductive tract pathogens. ${ }^{48}$ Another group studying the murine reproductive tract response to GBS in mice sustained in estrus observed increased levels of IL-17 in the murine reproductive tract 30 days after inoculation, although this difference was not significant. ${ }^{19}$ Even though this work was carried out in a different murine background with another strain of GBS, this corroborates our results that induction of IL-17 within the vaginal tract requires GBS persistence beyond several weeks.

In our model, only $\sim 40 \%$ of mice were able to clear CBJ 111 from the vaginal tract by 4 weeks, and these mice demonstrated increased IL-17A + cells compared with mice that remained colonized. The host and microbial factors controlling development of the IL-17 response to GBS remain unknown and require further investigation. However, our experiments with exogenous IL-17A treatment suggest that regardless of the source of IL-17A, it may contribute to the reduction of GBS vaginal colonization. Prolonged treatment of rIL-17 (2 weeks) resulted in enhanced clearance of GBS from the vaginal tract with $75 \%$ clearance observed in treated mice compared with $45 \%$ in controls, although this difference was not statistically significant (data not shown). We should note that this level clearance in control mice is consistent with the $50 \%$ clearance we observed at later time points in an earlier experiment (Figure 1c), and thus we do not believe that the diluent impacted these results. This information combined with further studies may be useful for developing pharmacologic interventions such as vaccine development for controlling GBS within the vaginal tract. Additionally, because strains A909 and $\mathrm{COH} 1$ were effectively cleared from the murine vaginal tract without the stimulation of an IL-17 response, future studies should also seek to identify additional host immune responses and presence or absence of GBS constituents that allow for shorter persistence within the vaginal tract.

In summary, we have continued to demonstrate that murine models of vaginal colonization can be correlated with in vitro human cells to further deepen our understanding of GBS-host interactions within the reproductive tract. GBS strains differentially engage host innate and adaptive immune responses, an element that combined with future work, will lead to successful elimination of GBS as a global concern of neonatal and even geriatric disease.

\section{METHODS}

Bacterial strains. Streptococcus agalactiae (GBS) wild-type clinical isolates A909 (serotype Ia), ${ }^{49} \mathrm{COH} 1$ (serotype III), ${ }^{50}$ and CJB111 (serotype V) (ATCC BAA-23, American Type Culture Collection, Manassas, VA) were grown aerobically in Todd-Hewitt broth (Hardy Diagnostics, Santa Maria, CA) at $37^{\circ} \mathrm{C}$.

Human cell lines. Immortalized human vaginal (VK2/E6E7), ectocervical (Ect1/E6E7), and endocervical (End1/E6E7) epithelial cell lines were acquired from American Type Culture Collection (ATCC CRL-2616, ATCC CRL-2614, and ATCC CRL-2615, respectively). Cell lines (passage 5-25) were cultured in keratinocyte serum-free medium (Life Technologies, Carlsbad, CA) with $0.5 \mathrm{ng} \mathrm{ml}^{-1}$ human recombinant epidermal growth factor and $0.05 \mathrm{mg} \mathrm{ml}^{-1}$ bovine pituitary extract at $37^{\circ} \mathrm{C}$ with $5 \% \mathrm{CO}_{2}$.

In vitro cell assays. GBS adherence, invasion, and intracellular survival assays of cell lines were conducted as described previously. ${ }^{12}$ Briefly, cells were grown to confluency in 24-well tissue culture plates and washed before bacterial infection. Bacteria were grown to mid-log phase and added at a multiplicity of infection (MOI) of 10 for adherence and invasion assays, and an MOI of 1 for intracellular survival assays to prevent the possibility of cellular toxicity over time. For adherence assays, after $2 \mathrm{~h}$ of incubation, cells were washed six times with PBS. Cells were lifted from plates by adding trypsin-EDTA and then lysed with $0.025 \%$ Triton X-100. Lysates were serially diluted and plated on Todd-Hewitt broth agar plates to quantify adherent CFUs. Total adherent CFU was calculated as (total CFU recovered/ total $\mathrm{CFU}$ of original inoculum) $\times 100 \%$. To quantify invading bacteria, cells were incubated with GBS for $2 \mathrm{~h}$, monolayers washed three times with PBS, treated with media containing antibiotics, and incubated for an additional $2 \mathrm{~h}$ for invasion assays, or as given for survival assays. Cells were washed three times with PBS, lysed as described above, and viable intracellular GBS determined by serial dilution plating as quantified above.

Reverse transcription (RT)-qPCR, ELISA, and western blot of cell lines. To quantify gene expression induction, human vaginal, ectocervical, and endocervical cells were grown to confluency in 24well tissue culture plates, washed before bacterial infection, and given fresh media. Bacteria were grown to mid-log phase, added to cells at an MOI of 10 , and incubated for $4 \mathrm{~h}$. Cells were lysed, then total RNA was extracted, and reverse transcription (Macherey-Nagel, Düren, Germany) and qPCR (quantitative PCR) were performed (Quanta Biosciences, Gaithersburg, MD). Human primer sequences used are as follows: GAPDH (glyceraldehyde 3-phosphate dehydrogenase)forward sequence, $5^{\prime}$-GAAGGTGAAGGTCGGAGTGAA-3' and reverse sequence, 5-TCCTGGAAGATGGTGATGGGA-3', IL-1 $\beta$ forward sequence $5^{\prime}$-GCCCTAAACAGATGAAGTGCTC- $3^{\prime}$ and reverse sequence, $5^{\prime}$-GAACCAGCATCTTCCTCAG-3' ; IL-6-forward 
sequence, $5^{\prime}$-GGAGACTTGCCTGGTGAAAA- $3^{\prime}$ and reverse sequence, $5^{\prime}$-CAGGGGTGGTTATTGCATCT- $3^{\prime}$; IL-23 ( $\alpha$-subunit)forward sequence, $5^{\prime}$-GCTTCAAAATCCTTCGCAG- $3^{\prime}$ and reverse sequence, $5^{\prime}$-TATCTGAGTGCCATCCTTGAG-3'; and IL-36 $\gamma-$ forward sequence, $5^{\prime}$-GAAACCCTTCCTTTTCTACCGTG-3' and reverse sequence, 5'-GCTGGTCTCTCTTGGAGGAG-3'.

For ELISA and western blot assays, human cell lines were infected as described above with several modifications. Bacteria were added at an MOI of 10 and cells were incubated with bacteria for 6-10 h, washed, and cell lysates were analyzed for cytokine production using human IL-1 $\beta$ ELISA Kit (R\&D Systems, Minneapolis, MN) according to the manufacturer's instructions. Cell lysates were also analyzed via western blot as described. ${ }^{13}$ Membranes were probed with antibodies against human GAPDH (1:150,000; EMD Millipore, Billerica, MA) or human IL-36 (1:400; R\&D Systems), and analyzed using ImageJ version $1.46 \mathrm{r}$ (National Institutes of Health, Bethesda, MD).

Mouse model of GBS vaginal colonization. All animal work was authorized by the Office of Lab Animal Care at San Diego State University and conducted using approved veterinary standards. The 8to 12-week-old female CD1 mice (Charles River Laboratories, Wilmington, MA) were injected intraperitoneally with $0.5 \mathrm{mg}$ $17 \beta$-estradiol (Sigma-Aldrich, St Louis, MO) in $100 \mu \mathrm{l}$ sesame oil on day $-1 .^{10,12}$ On day 0 , mice were vaginally inoculated with $1 \times 10^{7} \mathrm{CFU}$ GBS in $10 \mu \mathrm{l}$ of PBS (or PBS as a control for some experiments), and on subsequent days, each vaginal lumen was swabbed with a sterile ultrafine swab, and recovered GBS were enumerated by light pink or mauve colonies on CHROMagar StrepB agar (DRG International, Springfield, NJ). ${ }^{10}$

Tissue dissection, homogenization, and ELISA. For tissue collection, mice were killed using $\mathrm{CO}_{2}$ asphyxiation and reproductive tracts excised from mid-uterine horn to just proximal of the vulva. Tissues were visually separated by sterile razor blade to prevent bacterial crosscontamination between tissues. Tissues were placed in PBS with a protease inhibitor cocktail and homogenized with $1.0 \mathrm{~mm}$ zirconia beads using a Mini-Beadbeater (BioSpec Products, Bartlesville, OK). For initial screening, tissues were analyzed with the Mouse Proinflammatory Panel 1 V-PLEX Kit (Meso Scale Discovery, Rockville, MD) as per the manufacturer's instructions. ELISA assays were performed on tissue homogenates for KC and MIP-2 (R\&D Systems), as well as IL-1 $\beta$, IL-23, and IL-17 (eBioscience, San Diego, CA) as described by the manufacturer.

Tissue digestion and flow cytometry. Conversely, murine reproductive tract tissues were obtained as described above and subjected to enzymatic digestion. Tissues were finely diced with a sterile razor blade and incubated for $2 \mathrm{~h}$ at $37^{\circ} \mathrm{C}$ in RPMI- 1640 containing $10 \%$ fetal bovine serum, $0.4 \mathrm{mg} \mathrm{ml}^{-1}$ collagenase, and 1:1,000 brefeldin A (BD Biosciences, San Jose, CA). During this incubation, tissues were vigorously pipetted through $1,000 \mu \mathrm{l}$ and then $200 \mu \mathrm{l}$ pipette tips to aid in tissue digestion. Following digestion, samples were placed in fresh RPMI-1640 with 10\% FBS and 1:1,000 brefeldin A and incubated for an additional $4 \mathrm{~h}$ at $37^{\circ} \mathrm{C}$. Samples were surfaced-stained with fluorochrome-conjugated antibodies CD11b-PE and Ly6G-FITC clone 1A8 (BD Biosciences), as well as antibody CD4-PE-Cy7 (eBioscience). Samples were then fixed and permeabilized using BD Cytofix/Cytoperm (BD Biosciences) per manufacturer's instructions, and stained with IL-17-AlexaFluor647 (BD Biosciences). Before performing flow cytometry, samples were passed through $40 \mu \mathrm{m}$ filter tips to remove larger tissue debris. Samples were analyzed with an Accuri C6 Cytometer (BD Biosciences) and cell populations were assessed for the percent of fluorescent staining and staining brightness using Accuri analysis software (BD Biosciences).

Immunohistochemistry. Whole reproductive tract tissues were collected as described above and were fixed with paraformaldehyde and embedded in paraffin. For immunohistochemistry, sections were deparaffinized, rehydrated, and microwave heated in citrate buffer for antigen retrieval. Tissues were incubated with rabbit polyclonal anti-IL-17 (ab91649; Abcam, Cambridge, MA) at $5 \mu \mathrm{g} \mathrm{ml}^{-1}$ overnight, then incubated with goat anti-rabbit IgG-HRP, and developed with diaminobenzidine chromogen (Sigma-Aldrich). Tissues were counterstained with hematoxylin and visualized on a Zeiss upright microscope (Zeiss, Thornwood, NY) with attached Axiocam Icc3 camera (Zeiss) at $\times 200$ magnification.

Recombinant protein treatment. For rIL-17 treatment experiments, mice were first colonized with $1 \times 10^{7} \mathrm{CFU}$ of CJB111 as described above. One day later, mice were swabbed to determine colonization status, and were then treated with an intravaginal dose of 50-100 pg of recombinant mouse IL-17 (eBioscience) in $10 \mu \mathrm{l}$ of diluent, or only diluent as a control. Mice were swabbed and treated daily for the duration of the experiment.

Statistical analysis. GraphPad Prism version 5.04 was used for statistical analyses. Differences in recovered bacteria for intracellular survival, cytokine transcripts, and protein from in vitro assays were evaluated using two-way repeated-measures analysis of variance (ANOVA) with Bonferroni's multiple comparisons post-test. Oneway ANOVA with Bonferroni's multiple comparisons post-test was used for all other in vitro assays. In vivo results for recovered bacteria and ELISA experiments were analyzed using Kruskal-Wallis with Dunn's multiple comparisons post-test. In vivo flow cytometry and recombinant protein experimental data were analyzed with MannWhitney. Statistical significance was accepted at $P<0.05$.

\section{ACKNOWLEDGMENTS}

We thank the vivarium manager and staff at San Diego State University for support with animal husbandry. K.A.P. acknowledges the Inamori Foundation and the San Diego Chapter of the ARCS Foundation for support. K.S.D. was supported by an R01 NS051247, from the National Institutes of Health.

\section{DISCLOSURE}

The authors declare no conflict of interest.

c) 2015 Society for Mucosal Immunology

\section{REFERENCES}

1. Ohlsson, A. \& Shah, V.S. Intrapartum antibiotics for known maternal Group B streptococcal colonization. Cochrane Database Syst. Rev. 6, CD007467 (2014).

2. Le Doare, K. \& Kampmann, B. Breast milk and Group B streptococcal infection: vector of transmission or vehicle for protection? Vaccine $\mathbf{3 2}$, 3128-3132 (2014).

3. Centers for Disease Control and Prevention. Active Bacterial Core Surveillance Report, Emerging Infections Program Network, Group B Streptococcus, 2012 (2013)

4. Stoll, B.J. \& Schuchat, A. Maternal carriage of group B streptococci in developing countries. Pediatr. Infect. Dis. J. 17, 499-503 (1998).

5. Regan, J.A., Klebanoff, M.A. \& Nugent, R.P. The epidemiology of group B streptococcal colonization in pregnancy. Vaginal Infections and Prematurity Study Group. Obstet. Gynecol. 77, 604-610 (1991).

6. Verani, J.R., McGee, L. \& Schrag, S.J. Prevention of perinatal group B streptococcal disease-revised guidelines from CDC, 2010. Morb. Mort. Wkly. Rep. 59, 1-36 (2010).

7. Phares, C.R. et al. Epidemiology of invasive group B streptococcal disease in the United States, 1999-2005. JAMA 299, 2056-2065 (2008).

8. Falagas, M.E., Rosmarakis, E.S., Avramopoulos, I. \& Vakalis, N. Streptococcus agalactiae infections in non-pregnant adults: single center experience of a growing clinical problem. Med. Sci. Monit. 12, CR447CR451 (2006)

9. Park, S.E., Jiang, S. \& Wessels, M.R. CsrRS and environmental pH regulate group B streptococcus adherence to human epithelial cells and extracellular matrix. Infect. Immun. 80, 3975-3984 (2012). 
10. Patras, K.A. et al. Group B Streptococcus CovR regulation modulates host immune signalling pathways to promote vaginal colonization. Cell. Microbiol. 15, 1154-1167 (2013).

11. Wang, N.Y. et al. Group B streptococcal serine-rich repeat proteins promote interaction with fibrinogen and vaginal colonization. J. Infect. Dis. 210, 982-991 (2014).

12. Sheen, T.R. et al. Serine-rich repeat proteins and pili promote Streptococcus agalactiae colonization of the vaginal tract. J. Bacteriol. 193, 6834-6842 (2011).

13. Banerjee, A. et al. Bacterial Pili exploit integrin machinery to promote immune activation and efficient blood-brain barrier penetration. Nat. Commun. 2, 462 (2011).

14. Schubert, A. et al. A fibrinogen receptor from group B Streptococcus interacts with fibrinogen by repetitive units with novel ligand binding sites. Mol. Microbiol. 46, 557-569 (2002).

15. Tamura, G.S. \& Rubens, C.E. Group B streptococci adhere to a variant of fibronectin attached to a solid phase. Mol. Microbiol. 15, 581-589 (1995).

16. Franken, C. et al. Horizontal gene transfer and host specificity of betahaemolytic streptococci: the role of a putative composite transposon containing scpB and Imb. Mol. Microbiol. 41, 925-935 (2001).

17. Zegels, G., Van Raemdonck, G.A., Coen, E.P., Tjalma, W.A. \& Van Ostade, X.W. Comprehensive proteomic analysis of human cervical-vaginal fluid using colposcopy samples. Proteome Sci. 7, 17 (2009).

18. Soares, G.C. et al. Metallopeptidases produced by group B Streptococcus: influence of proteolytic inhibitors on growth and on interaction with human cell lineages. Int. J. Mol. Med. 22, 119-125 (2008).

19. Carey, A.J. et al. Infection and cellular defense dynamics in a novel 17betaestradiol murine model of chronic human group B streptococcus genital tract colonization reveal a role for hemolysin in persistence and neutrophil accumulation. J. Immunol. 192, 1718-1731 (2014).

20. Randis, T.M. et al. Group B Streptococcus beta-hemolysin/cytolysin breaches maternal-fetal barriers to cause preterm birth and intrauterine fetal demise in vivo. J. Infect. Dis. 210, 265-273 (2014).

21. Faralla, C. et al. Analysis of two-component systems in group B Streptococcus shows that RgfAC and the novel FspSR modulate virulence and bacterial fitness. mBio 5, e00870-00814 (2014).

22. Yow, M.D. et al. The natural history of group B streptococcal colonization in the pregnant woman and her offspring. I. Colonization studies. Am. J. Obstet. Gynecol. 137, 34-38 (1980).

23. Bodaszewska-Lubas, M. et al. Adherence of group B streptococci to human rectal and vaginal epithelial cell lines in relation to capsular polysaccharides as well as alpha-like protein genes-pilot study. Pol. J. Microbiol. 62, 85-90 (2013).

24. Mei, J. et al. Cxcr2 and Cxcl5 regulate the IL-17/G-CSF axis and neutrophil homeostasis in mice. J. Clin. Invest. 122, 974-986 (2012).

25. Iwakura, Y. \& Ishigame, H. The IL-23/IL-17 axis in inflammation. J. Clin. Invest. 116, 1218-1222 (2006).

26. Pietrella, D. et al. Th17 cells and IL-17 in protective immunity to vaginal candidiasis. PLoS One 6, e22770 (2011).

27. Feinen, B., Jerse, A.E., Gaffen, S.L. \& Russell, M.W. Critical role of Th17 responses in a murine model of Neisseria gonorrhoeae genital infection. Mucosal Immunol. 3, 312-321 (2010).

28. Berti, F. et al. Structure of the type IX Group B streptococcus capsular polysaccharide and its evolutionary relationship with types Vand VII. J. Biol. Chem. 289, 23437-23448 (2014).

29. Baker, C.J. \& Barrett, F.F. Group B streptococcal infections in infants. The importance of the various serotypes. JAMA 230, 1158-1160 (1974).

30. Tettelin, $\mathrm{H}$. et al. Genome analysis of multiple pathogenic isolates of Streptococcus agalactiae: implications for the microbial 'pan-genome'. Proc. Natl. Acad. Sci. USA 102, 13950-13955 (2005).
31. Rinaudo, C.D. et al. Specific involvement of pilus type 2a in biofilm formation in group B Streptococcus. PLoS One 5, e9216 (2010).

32. Baker, C.J. et al. Immune response of healthy women to 2 different group B streptococcal type $\mathrm{V}$ capsular polysaccharide-protein conjugate vaccines. J. Infect. Dis. 189, 1103-1112 (2004).

33. Nikolaitchouk, N., Andersch, B., Falsen, E., Strombeck, L. \& MattsbyBaltzer, I. The lower genital tract microbiota in relation to cytokine-, SLPIand endotoxin levels: application of checkerboard DNA-DNA hybridization (CDH). APMIS 116, 263-277 (2008).

34. Mitchell, K. et al. Group B Streptococcus colonization and higher maternal IL-1 beta concentrations are associated with early term births. J. Matern. Fetal Neonat. Med. 26, 56-61 (2013).

35. van de Veerdonk, F.L. et al. Th17 responses and host defense against microorganisms: an overview. BMB Rep. 42, 776-787 (2009).

36. Buonocore, S. et al. Innate lymphoid cells drive interleukin-23-dependent innate intestinal pathology. Nature 464, 1371-1375 (2010).

37. Vigne, S. et al. IL-36R ligands are potent regulators of dendritic and Tcells. Blood 118, 5813-5823 (2011).

38. Fichorova, R.N. \& Anderson, D.J. Differential expression of immunobiological mediators by immortalized human cervical and vaginal epithelial cells. Biol. Reprod. 60, 508-514 (1999).

39. Mossop, H., Linhares, I.M., Bongiovanni, A.M., Ledger, W.J. \& Witkin, S.S. Influence of lactic acid on endogenous and viral RNA-induced immune mediator production by vaginal epithelial cells. Obstet. Gynecol. 118 , 840-846 (2011).

40. Fanibunda, S.E., Modi, D.N. \& Bandivdekar, A.H. HIV gp120 induced gene expression signatures in vaginal epithelial cells. Microbes Infect. 15, 806-815 (2013).

41. Ye, P. et al. Requirement of interleukin 17 receptor signaling for lung $C X C$ chemokine and granulocyte colony-stimulating factor expression, neutrophil recruitment, and host defense. J. Exp. Med. 194, 519-527 (2001).

42. Edwards, L.A. et al. Delineation of the innate and adaptive T-cell immune outcome in the human host in response to Campylobacter jejuni infection. PLoS One 5, e15398 (2010).

43. Ivanov, I.I. et al. Specific microbiota direct the differentiation of IL-17producing T-helper cells in the mucosa of the small intestine. Cell Host Microbe 4, 337-349 (2008).

44. Kirjavainen, P.V. et al. Abnormal immunological profile and vaginal microbiota in women prone to urinary tract infections. Clin. Vaccine Immunol. 16, 29-36 (2009).

45. Kubota, T., Nojima, M. \& Itoh, S. Vaginal bacterial flora of pregnant women colonized with group B streptococcus. J. Infect. Chemother. 8, 326-330 (2002).

46. Ferretti, S., Bonneau, O., Dubois, G.R., Jones, C.E. \& Trifilieff, A. IL-17, produced by lymphocytes and neutrophils, is necessary for lipopolysaccharide-induced airway neutrophilia: $\mathrm{IL}-15$ as a possible trigger. J. Immunol. 170, 2106-2112 (2003).

47. Zhang, Z., Clarke, T.B. \& Weiser, J.N. Cellular effectors mediating Th17dependent clearance of pneumococcal colonization in mice. J. Clin. Invest. 119, 1899-1909 (2009).

48. Lieberman, J.A., Moscicki, A.B., Sumerel, J.L., Ma, Y. \& Scott, M.E. Determination of cytokine protein levels in cervical mucus samples from young women by a multiplex immunoassay method and assessment of correlates. Clin. Vaccine Immunol. 15, 49-54 (2008).

49. Madoff, L.C., Michel, J.L. \& Kasper, D.L. A monoclonal antibody identifies a protective C-protein alpha-antigen epitope in group B streptococci. Infect. Immun. 59, 204-210 (1991).

50. Wilson, C.B. \& Weaver, W.M. Comparative susceptibility of group B streptococci and Staphylococcus aureus to killing by oxygen metabolites. J. Infect. Dis. 152, 323-329 (1985). 